\title{
2,3,7,8-Tetrachlorodibenzo- $p$-dioxin affects fluctuating asymmetry of molar shape in mice, and an epistatic interaction of two genes for molar size
}

\author{
JM Keller, DE Allen ${ }^{1}$, CR Davis ${ }^{2}$ and LJ Leamy \\ Department of Biology, University of North Carolina at Charlotte, Charlotte, NC, USA
}

Fluctuating asymmetry (FA), random variation between left and right sides in a bilaterally symmetrical character, is a commonly used measure of developmental instability that is expected to increase with increasing environmental stress. One potential stressor is 2,3,7,8-tetrachlorodibenzo- $p$-dioxin (TCDD), a powerful toxicant known to disturb tooth development. In this study, mice in the $F_{2}$ generation produced from an intercross between two inbred strains (C57BL/6J and $\mathrm{AKR} / \mathrm{J}$ ) were exposed in utero to TCDD. We hypothesized that TCDD would increase FA in the molars of exposed mice over that of the control mice. In addition, we hypothesized that we would discover genes for molar size, shape or asymmetry whose expression would be affected by TCDD. We detected a very small, but significant, increase in FA of

Keywords: TCDD; fluctuating asymmetry; molars; QTL

\section{Introduction}

Developmental stability (DS) is the ability of a particular genotype to produce a consistent phenotype in a particular environment despite random perturbations during development (Palmer, 1994). Thus reduced DS, or increased developmental instability (DI), results in a greater deviation from the expected phenotype within a given environment (Palmer, 1994). A common measure of DI is fluctuating asymmetry (FA), or variation in the unsigned differences between the left and the right sides of a bilateral trait (Palmer, 1994). It has been proposed that environmental toxins and pollutants may disrupt development and therefore increase FA (Palmer, 1994). This result has been supported by many (see Leamy and Klingenberg, 2005), although not all, studies (Allen and Leamy, 2001; Davis et al., 2002).

One reason for the popularity of FA as an indicator of environmental stress has been its presumed origin from random developmental variation (Palmer, 1994; Palmer and Strobeck, 2003). A number of genetical studies of FA

Correspondence: Dr LJ Leamy, Department of Biology, University of North Carolina at Charlotte, Charlotte, NC 28223, USA.

E-mail: ljleamy@email.uncc.edu

${ }^{1}$ Current address: Institute of Evolutionary Biology, University of Edinburgh, EH9 3JT, UK

${ }^{2}$ Current address: Department of Biochemistry and Molecular Biology, University of South Florida, Tampa, FL 33612, USA

Received 22 February 2006; revised 16 June 2006; accepted 23

November 2006; published online 10 January 2007 molar shape (but not size) in the TCDD-exposed mice compared to the control mice, although molar size and shape did not differ between these groups. Although we did not uncover any genes that acted differently in the TCDD exposed and control groups, we did identify two genes whose dominance by additive epistatic effect on molar size was affected by TCDD. We concluded that although TCDD may be affecting the expression of some genes governing the development of molars in our population of mice, FA of molar size and shape is not a particularly sensitive indicator of this effect.

Heredity (2007) 98, 259-267. doi:10.1038/sj.hdy.6800928; published online 10 January 2007

have shown that its heritability is typically very low or zero (Leamy and Klingenberg, 2005) and have detected few quantitative trait loci (QTL) for FA (Klingenberg et al., 2001; Davis et al., 2002; Leamy and Klingenberg, 2005). However, some mutations have been found that increase FA (Leamy et al., 2001; Leamy and Klingenberg, 2005). It is also possible that FA in a given trait may be affected by one or more genes responsible for the normal development of that trait (Klingenberg and McIntyre, 1998), especially in a stressful environment (Leamy and Klingenberg, 2005). In fact, Davis et al. (2002) recently discovered a QTL for mandible shape whose effects on FA of mandible shape differed depending on whether or not the mice were maternally exposed to $2,3,7,8$ tetrachlorodibenzo- $p$-dioxin (TCDD). This was despite the fact that no QTL for FA itself was found (Davis et al., 2002) and there was no significant difference detected in mandible size or shape FA between treated and control mice in the same population (Allen and Leamy, 2001).

TCDD is a potent environmental toxicant that is released during high temperature combustion common in certain industries (Birnbaum, 1994). TCDD is found naturally in animal fat and can bioaccumulate in animal tissues such as those in the mammary glands (Birnbaum, 1994; Partanen et al., 1998). Most effects of TCDD occur when it binds to the aryl hydrocarbon receptor (AHR), a ligand-activated transcriptional regulator controlled by the Ahr locus (Birnbaum, 1994). Once TCDD is bound to $\mathrm{AHR}$, this complex migrates to the nucleus where it dimerizes with the AHR nuclear translocator, or ARNT. 
The ligand-AHR-ARNT complex is capable of binding to a number of dioxin responsive elements on DNA upstream from several genes, altering their affinity for transcription factors and thus their rate of transcription (Whitlock, 1999). As a consequence, even very low (nontoxic) levels of TCDD produce developmental deformities ranging from cleft palate to kidney abnormalities and gonadal atrophy (Birnbaum, 1995). Although the action of TCDD appears to be species-, strain- and even tissuespecific (Birnbaum, 1995), tooth development is particularly sensitive to its effects in humans, rats and mice (Alaluusua et al., 1993; Partanen et al., 1998).

The population of mice analyzed by Allen and Leamy (2001) and Davis et al. (2002) seemed ideal for a followup study aimed at comparing FA in tooth traits in the treated and control groups. Although Allen and Leamy (2001) did not detect differences in mandible FA in mice exposed versus those not exposed to TCDD, it seemed worthwhile to test for the effects of TCDD on molar FA. Our working hypothesis was that the in utero exposure of these mice to TCDD would increase FA in molar size and shape over that for mice not exposed to TCDD. In addition, based on the work by Davis et al. (2002), we hypothesized that we would find genes whose expression on molar size, shape or asymmetry would be affected by TCDD.

\section{Materials and methods}

\section{Population}

The mice evaluated in this study were members of the $\mathrm{F}_{2}$ generation derived from an intercross between the AKR/ J and C57BL/6J inbred strains of mice (Allen and Leamy, 2001). The study population consisted of the offspring of pregnant $F_{1}$ mothers that received one of four different treatments on gestation day 9 (GD9, where GD0 was indicated by the presence of a vaginal plug). One treatment group (T1) consisted of mice reared from $F_{1}$ mothers who received a dose of $1 \mu \mathrm{g}$ TCDD $/ \mathrm{kg}$ body weight whereas the second treatment group (T2) consisted of mice whose mothers received $0.5 \mu \mathrm{g}$ TCDD/kg body weight. Both treatments were delivered in a toluene/corn oil vehicle via oral gavage. The first control group (C1) was reared from mothers who received a dose of toluene/corn oil equivalent to that given to mice in the T1 group. The second control group (C2) received no treatment (Allen and Leamy 2001).

The TCDD dosages ( 1 and $0.5 \mu \mathrm{g} / \mathrm{kg}$ ) were chosen because doses as low as $1 \mu \mathrm{g}$ TCDD/kg caused kidney nephrosis in mice (Moore et al., 1973) but were otherwise non-toxic (Allen and Leamy, 2001). Allen and Leamy (2001) hypothesized that even such low doses of TCDD would influence the development of asymmetry in mouse mandibles. The $F_{1}$ mothers were dosed on GD9 because bone development begins at that time in mice (Kaufman, 1992). This dosing schedule also seemed appropriate for evaluating the effects of TCDD on molar traits because the first signs of tooth development in mice are visible on GD11 (Cobourne and Sharpe, 2003).

All $\mathrm{F}_{2}$ offspring produced from these matings were weaned at 19 days of age and were separated by sex at 30 days of age (Allen and Leamy, 2001). At 60 days, approximately five mice from each litter were weighed, killed and skeletonized using dermestid beetles. In addi- tion, a tail clip from each mouse was stored at $-80^{\circ} \mathrm{C}$ for DNA extraction and microsatellite genotyping. After eliminating individuals with missing or broken jaws or teeth, approximately 100 mice per group remained $(n=415)$. Each group contained approximately equal numbers of males and females. All procedures involving the rearing and treatment of animals were approved by the Institutional Animal Care and Use Committee at the University of North Carolina at Charlotte.

\section{Genotyping}

Previously, Davis et al. (2002) extracted DNA from tail clips of mice in this population and genotyped two microsatellite loci, D12Mit112 and D11Mit258. D12Mit112 was chosen because it is closely linked to the Ahr locus on chromosome 12, the locus that produces AHR to which TCDD binds and initiates the signaling pathway resulting in TCDD's manifold effects (Birnbaum, 1994). D11Mit258 was chosen because it is located on chromosome 11 near a QTL previously found to affect mandible development (Klingenberg et al., 2001). Samples of the DNA from this population were stored at $-20^{\circ} \mathrm{C}$ and were available for use in this investigation.

We chose three additional polymorphic microsatellites on chromosome 12 (D12Mit214, D12Mit105 and D12Mit101) that, when combined with the existing data for D12Mit112, gave adequate coverage for a QTL scan of this chromosome. Each mouse was genotyped at each marker to determine if the individual was homozygous for $\mathrm{AKR} / \mathrm{J}(\mathrm{AA})$ or $\mathrm{C} 57 \mathrm{BL} / 6 \mathrm{~J}(\mathrm{BB})$ alleles, or heterozygous $(A B)$. The microsatellite regions were amplified using the standard protocols and primers supplied by Research Genetics (Huntsville, AL, USA). The resulting polymerase chain reaction (PCR) products were separated and visualized using $2.5 \%$ agarose gel electrophoresis and ethidium bromide staining. Although some of the data were unavailable owing to missing DNA samples or PCR products that could not be resolved on the gel, an adequate sample size $(n=396-397)$ was obtained for all three polymorphic microsatellite loci.

\section{Molar size and shape traits}

Left and right molar rows in each mouse were magnified using an Olympus SZ60 microscope with a Hitachi KPC550 video camera attached so that the image could be viewed on a computer monitor. The mandibles were placed upright to view the dorsal surface of the molar row for image capture. A total of nine landmark points on each molar row (Figure 1) were digitized via the Measurement $T V$ program. The nine landmarks yielded a total of 18 coordinate $(x, y)$ values for both left and right mandibular molar rows. After completing a single round

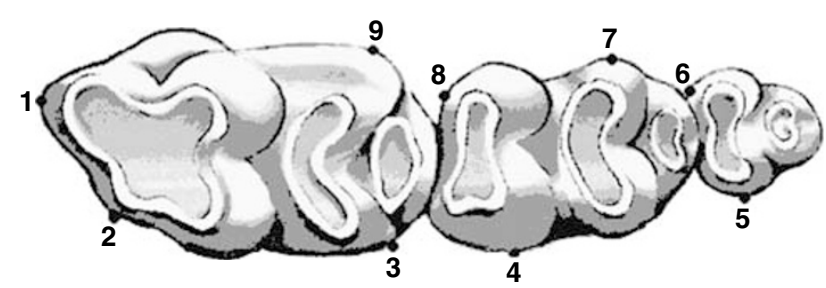

Figure 1 Locations of the nine landmarks digitized on the mandibular molar rows. 
of measurements on the entire sample, a second set of coordinates was recorded.

The 18 coordinates obtained from the digitizing process were used to create molar size and shape traits. The centroid size (the square root of the sum of the squared distances between each landmark point and the mean of the $x$ and $y$ coordinates of all landmark points) was our measure of the size of each molar row. In addition, 18 shape variables were calculated using the Procrustes method, a four-step process that reflects one molar row of a pair and then scales, superimposes and rotates each image to obtain the best fit between the right and left sides of an individual (see Klingenberg and McIntyre, 1998). This process reduces the number of degrees of freedom by four, which for our sample, resulted in a total of 14 (18-4) Procrustes degrees of freedom. Because these shape variables collectively define a multivariate shape trait, variation in any one of the coordinates is only one part of the change in the entire shape, and shape is therefore best expressed using figures which depict the magnitude and direction of change at each of the landmarks (Klingenberg and McIntyre, 1998). The mean of the left and right sides of each individual was calculated for centroid size and all 18 shape variables for use in assessment of molar size and shape differences.

\section{Assessment of asymmetry and measurement error}

The existence of significant molar size asymmetry was assessed using a mixed model two-way analysis of variance (ANOVA) (Palmer, 1994). In this model, the random factor, individuals (I), is used to assess the variation among mice (d.f. $=n-1$ where $n$ is the number of mice). The fixed factor, sides (S), assesses directional asymmetry (DA) (the presence of a consistent difference between the two sides of a bilateral trait; d.f. $=1$ ). The interaction $(\mathrm{I} \times \mathrm{S} ; n-1$ d.f.) evaluates the presence of FA. The error assesses the difference between replicate measures (Palmer, 1994). Mean squares (MSs) for individuals and for the interaction were tested over the error MS but the MS for sides was tested using the interaction MS (Sokal and Rohlf, 1995). A significant interaction indicates that FA is greater than can be explained by measurement error alone. In order to assess the contribution of each random factor to the total variation seen in molar centroid size, variance components also were calculated for individual, individual $\times$ side and error (Palmer, 1994; Davis et al., 2002).

Although molar size traits were evaluated using a conventional ANOVA, molar shape was evaluated using a Procrustes ANOVA (Klingenberg and McIntyre, 1998; Davis et al., 2002). The sums of squares in a Procrustes ANOVA are equal to the sum of the individual sums of squares for each of the (18) multivariate shape variables and the degrees of freedom are equal to the degrees of freedom of each trait multiplied by the Procrustes degrees of freedom, or 14 in this case (Allen and Leamy, 2001; Klingenberg et al., 2001). The variance components for molar shape were calculated from the means of the variance components for the 18 individual shape variables.

\section{Molar asymmetry traits}

The means of the two replicate measures of each of the 19 variables (centroid size and the 18 shape variables) calculated above were used to create FA values for both the size and the shape of the molar row. To accomplish this, we first standardized the mean of the left minus right side differences $(\mathrm{L}-\mathrm{R})$ in each of the four groups to zero (Palmer, 1994) to adjust for any DA present. As the $(\mathrm{L}-\mathrm{R})$ values of all groups exhibited normal distributions, no antisymmetry was present in the population and these differences within each group were attributable to FA (Palmer, 1994). After correcting for DA, FA of molar size was calculated as the absolute value of the difference between the right and left values for centroid size (Palmer, 1994). In order to evaluate potential scaling effects on FA, the values obtained for molar size FA were regressed on molar centroid size. No significant scaling effects were detected and thus no further adjustments were made for our measure of molar size FA.

A single measure of FA of molar shape was calculated using a new method suggested by Klingenberg and Monteiro (2005); see also Leamy et al. (2005). First, we obtained the signed differences of sides (L-R) for all 18 Procrustes values, and adjusted them for DA as before. We then ran a principal components analysis of the covariances of these differences, and standardized the component scores for the 14 components with non-zero eigenvalues by dividing each score by the square root of its appropriate eigenvalue. Finally, molar shape FA values were calculated for each mouse from the square root of the sums of squares of these standardized component scores. This approach allowed us to employ univariate rather than multivariate statistical methods for the analysis of shape FA. For purposes of comparison, an alternate measure of shape FA was calculated by Procrustes distances obtained for each individual from the square root of the sums of squares of the differences between left and right sides of the 18 Procrustes shape variables.

\section{Statistical adjustments}

Before the assessment of the effects of environment (TCDD) and genotype on the molar characters, potential differences owing to sex and litter size were evaluated. No significant effects owing to litter size were detected, so this variable was eliminated from the analysis. However, sex significantly affected molar centroid size (although no sex by genotype interactions were detected). To simplify the final statistical analysis, therefore, we eliminated differences between the two sexes for centroid size by adding the residuals from a regression to the grand mean of centroid size.

As was performed previously (Allen and Leamy, 2001; Davis et al., 2002), we used orthogonal comparisons to test both for differences in the molar traits between the two treatment groups and for differences between the two control groups. We used a mixed-model ANOVA in which group was a fixed factor and litter (nested within group) was a random factor. Litter was included in these analyses to adjust for possible differences among litters caused by maternal effects or other environmental factors. Owing to its multivariate nature, molar shape was evaluated using a multivariate ANOVA (MANOVA) of the same design. The sequential Bonferroni procedure was used to evaluate significance where required to keep the experiment-wise error rate at 5\% (Sokal and Rohlf, 1995). No significant differences between the two control 
groups or between the two treatment groups were detected. Because of this, we were able to pool groups, forming a single treatment group and a single control group, and test for differences between these two groups using the same ANOVA (univariate traits) or MANOVA (shape) model just described (see also Allen and Leamy, 2001).

\section{Single marker analysis of chromosome 11}

A mixed-model ANOVA (Sokal and Rohlf, 1995) was used to evaluate single marker (D11Mit258) genotypic effects and environmental (TCDD) effects on molar size and FA of molar size and shape. In this model, environment is a fixed effect with two levels (1 d.f.; treatment and control), genotype is a fixed effect associated with the three possible genotypes at this locus ( 2 d.f.; $\mathrm{AA}, \mathrm{AB}$ and $\mathrm{BB}$ ), and litter is a random effect nested within environment. A significant genotype effect is suggestive of a QTL located near D11Mit258. A significant environment effect indicates that the treatment and control groups differ and thus that TCDD is affecting the development of the trait. For FA of molar size and shape, environmental effects were evaluated for significance with one-tailed tests as it was hypothesized that FA would increase in the TCDD-treated group, although we also noted results for two-tailed tests (see below). A significant genotype by environment interaction implies that the effect of the QTL associated with D11Mit258 depends on whether or not TCDD was present. A significant litter effect indicates that there are differences among litters within the environments. Environment effects were tested using litter as the error term, but all other factors were tested over the error. A MANOVA of the same design was used to evaluate molar shape.

\section{QTL mapping and analysis}

We used interval mapping procedures (Haley and Knott, 1992) to search for QTL on chromosome 12 (C12). To accomplish this, additive genotypic index values of 1,0 , -1 and dominance genotypic index values of $-0.5,+0.5$, -0.5 were assigned to the three different genotypes (AA, $\mathrm{AB}, \mathrm{BB}$, respectively) at each of the four markers on $\mathrm{C} 12$. Map distances between markers were determined using Mapmaker 3.0 (Lincoln et al., 1992) and genotypic index values were calculated (Haley and Knott, 1992) at $2 \mathrm{cM}$ intervals between markers on $\mathrm{C} 12$. Associations between the additive and dominance genotypic index values at each location and each of the molar traits were assessed using a canonical correlation procedure (Leamy et al., 1999 b). Probabilities from these associations were used to calculate likelihood of odds (LOD) scores for each $2 \mathrm{cM}$ location across C12 (Leamy et al., 1999b). The highest LOD score for each molar trait was considered significant if it exceeded a calculated threshold value (Cheverud, 2001). The highest significant LOD score was taken to be suggestive of a QTL at that chromosomal location. Support intervals for each QTL were determined by a 1.0 drop in LOD scores on either side of the putative QTL (Lynch and Walsh, 1998).

Once a single QTL was found, a two-QTL model was used to test for the presence of a second QTL on C12. In this model, canonical correlations were used to assess the association between the molar trait and the additive and dominance genotypic values for every possible pair of locations on C12. The difference between the highest $\chi^{2}$ value obtained using the one QTL model and the highest $\chi^{2}$ value obtained using the two QTL model was compared to the critical $\chi^{2}$ value for 2 d.f. for centroid size or 28 d.f. for shape. If the difference between the two models exceeded the critical value, two QTL were presumed to be present (Leamy et al., 1999b).

Additive and dominance genotypic values of any QTL located were estimated from the partial regression coefficients calculated via multiple regressions of each molar trait on the genotypic index values at that site (Haley and Knott, 1992). The additive genotypic value (a) at each locus is equal to one half the difference between the means of the two homozygotes $((\mathrm{AA}-\mathrm{BB}) / 2)$, and the dominance genotypic value $(d)$ for each locus is equal to the difference between the mean of the heterozygotes and the midpoint of the two homozygotes $(\mathrm{AB}-(\mathrm{AA}+\mathrm{BB}) / 2)$. For the univariate traits, these regressions produce estimates of the $a$ and $d$ values, their standard errors and the percentage of variation explained by the QTL. However, for shape, the regression results in a series of vectors containing $18 a$ and $d$ values that possess both magnitude and direction. These vectors were used to calculate additive $(\|a\|)$ and dominance $(\|d\|)$ shape effects in units of Procrustes distance (Klingenberg et al., 2001). Multivariate regression of the shape traits on the additive and dominance index values was used to evaluate the significance of these shape effects. The effects of shape QTL were visualized using diagrams which depict the magnitude and direction of change in shape at each landmark (e.g., see Workman et al., 2002).

Gene-by-environment interactions were tested using canonical correlation of each of the molar traits with the interactions between the environment and the additive and dominance genotypic values, partialing the effects of the environment and the two genotypic scores. LOD scores were calculated for all interactions, and evaluated for significance using the same threshold value described previously. A significant LOD score from this analysis indicates that TCDD exposure affects the expression (additive or dominance effects) of a QTL in that region of chromosome 12 .

\section{Analysis of epistatic effects}

To test for epistatic effects between any QTL discovered on chromosomes 11 and 12, D11Mit258 genotypic index values were merged with the genotypic index values for the QTL on C12 and the molar phenotypic data. Using the canonical correlation approach described by Wolf et al. (2005), correlations of the molar traits with the four independent interactions of additive and dominance genotypic index values (additive by additive (aa), additive by dominance $(a d)$, dominance by additive $(d a)$ and dominance by dominance $(d d)$ ) were calculated, partialing out the four main effects (additive and dominance index values for both QTL). The significance of epistasis for the molar traits was indicated by the probabilities associated with $F$ approximations to the Wilks Lamba statistic generated from the canonical correlation analyses. We used the conventional 5\% significance level without adjustment for multiple comparisons problems as the QTL for epistasis testing 
were chosen without any knowledge of potential epistatic effects (see Leamy et al., 2005). Multiple regression was also used to estimate the four genotypic epistatic terms ( $a a, a d$, $d a$ and $d d)$ for each QTL pair. Epistasis was considered significant if any of the $t$-tests for these individual epistatic components reached the $0.5 / 4=0.0125$ probability level even if the $F$ test for overall epistasis was not significant.

If a significant epistatic interaction was found, we tested whether this interaction differed in the treatment and control groups using canonical correlation of that molar trait with the three-way interactions of the index scores for the C11 and C12 QTL and the environment, partialing out all main effects. The conventional 5\% level of significance was used to test whether the effect of the interaction between QTL on C11 and C12 affecting the molar trait differed depending on whether or not TCDD was present.

\section{Results}

\section{Preliminary analysis}

The results of the two-way ANOVAs for the replicate measures of molar centroid size and molar shape showed that the largest contribution to the total variation in each case was due to differences among individuals (78\% for molar centroid size, $43 \%$ for molar shape). MSs for sides also significantly influenced both molar size and shape, suggesting that some DA exists in both of these molar traits. More importantly, both interaction MSs were highly significant, indicating that FA exists for molar centroid size and molar shape. FA contributed approximately $11 \%$ of the size variation and almost $23 \%$ of the variation in shape. Measurement error was assessed by the variation between the replicate measures and accounted for approximately $11 \%$ of the variation in molar size and $35 \%$ of the variation in molar shape.

Table 1 lists the means and standard deviations for molar centroid size and FA of molar centroid size and shape for the pooled treatment and the pooled control groups. Centroid size does not differ significantly between groups, with each group having a mean centroid size of approximately $2.81 \mathrm{~mm}$. Similarly, MANOVA results showed that molar shape did not differ significantly between groups. The means of centroid size FA are similar for both groups (Table 1) and do not differ significantly. However, shape FA does differ significantly between groups $(P=0.019$ in a onetailed test, $P=0.038$ in a two-tailed test), with the mean of the treatment group (3.74) slightly exceeding the mean

Table 1 Means and s.d. for molar centroid size and FA of centroid size and shape

\begin{tabular}{llllll}
\hline & \multicolumn{2}{c}{ Treatment } & & \multicolumn{2}{c}{ Control } \\
\cline { 2 - 3 } & \multicolumn{1}{c}{ Mean } & s.d. & & Mean & s.d. \\
\hline Centroid size & $2.810 \mathrm{NS}$ & 0.048 & & 2.814 & 0.043 \\
Centroid size FA & $0.024 \mathrm{NS}$ & 0.015 & & 0.026 & 0.017 \\
Shape FA & $3.743^{*}$ & 0.809 & & 3.568 & 0.704 \\
\hline
\end{tabular}

Abbreviations: FA, fluctuating asymmetry; NS, nonsignificant; s.d., standard deviation.

${ }^{*} P<0.05$. of the control group (3.57). The mean for the Procrustes distance measure of shape FA also was higher in the treatment group (0.32) compared to the control group (0.30), and the difference between these two means was statistically significant ( $P=0.035$ in a one-tailed test). In summary, TCDD has acted to cause a small, but statistically significant, increase in FA of molar shape, although it apparently has not affected any of the other molar traits.

\section{C11 analysis}

ANOVA using the C11 marker and the environment as factors showed that the C11 genotype was not significant for any of the univariate molar traits (molar centroid size and FA of molar size and shape). This suggests that there is no QTL near the D11Mit258 marker affecting any of these traits. Environmental (TCDD) effects showed the same pattern as already seen in Table 1: significance for shape FA but not for molar centroid size or FA of centroid size. The genotype $\times$ environment interaction did not reach significance for any of these molar traits, although there was a highly significant litter effect on molar centroid size $(P=0.003)$.

The results of the MANOVA for molar shape indicated a highly significant $(F=3.37$, d.f. $=14, P<0.01)$ difference in molar shape among the D11Mit258 genotypes, suggesting that there is a QTL somewhere on C11 (QTL-SH11) that influences molar shape. There were no significant effects owing to environment or the genotype $\times$ environment interaction however, thus there is no evidence that TCDD affects the expression of QTL-SH11 on molar shape. The overall additive $(\|a\|)$ and dominance effects $(\|d\|)$ of $Q T L-S H 11$ were calculated in units of Procrustes distance as described previously, with additive effects being positive $(0.00421)$ and greater than dominance effects (0.00336).

Figure 2 depicts changes in the relative positions of all landmarks owing to QTL-SH11 as bars drawn from the mean of the landmark to the location of the mean plus the additive (or dominance) effect. Because of the subtlety of the changes, the effects have been multiplied by a factor of 25. The additive effects of this QTL appear to be acting primarily on the medial/lateral orientation of the molar row, particularly at landmarks 3, 6, 8 and 9 . Landmarks 3 and 9 are being shifted laterally, whereas landmarks 6 and 8 are shifted medially. The dominance effects appear to be spread more uniformly across the molar row. However, the greatest effects are still seen on the second and third molars, specifically at the positions of landmarks 4, 5, 6 and 7. As with additive effects, the medial/lateral orientation of the landmarks appears to be most affected. Landmarks 4 and 7 are shifted laterally, whereas landmarks 5 and 6 are shifted medially.

\section{C12 analysis}

For chromosome 12, no QTL were detected for FA of molar shape or FA of molar centroid size. However, the LOD score (3.606) associated with a QTL for molar centroid size reached $1 \%$ chromosome-wise significance (Table 2). This QTL, designated QTL-CS12, accounts for about $4.21 \%$ of the variation in molar size. Alleles at this locus exhibit significant additive effects $(a=0.0144$, $P<0.001 ; d=-0.0004, P>0.05)$, with the AKR $/ \mathrm{J}$ allele increasing molar centroid size. 


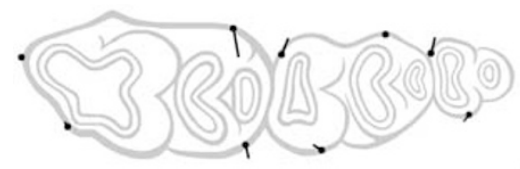

$a$

QTL-SHI1
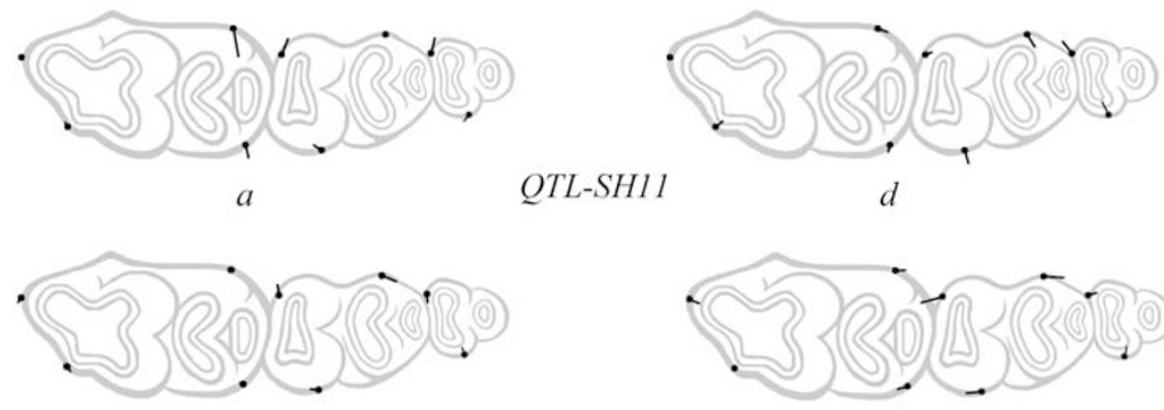

$a$

OTL-SHI2

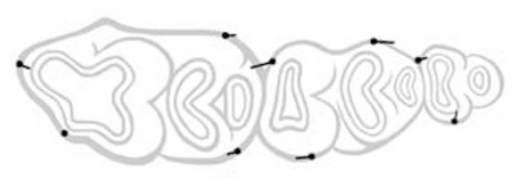

$d$

Figure 2 Additive and dominance effects of QTL-SH11 and QTL-SH12 on molar shape. Changes in the relative positions of all landmarks owing to each QTL are depicted as bars drawn from the mean of the landmark to the location of the mean plus the additive or dominance effect. Additive effects $(a)$ are shown in the left column whereas dominance effects $(d)$ are shown in the right column. All effects are scaled by a factor of $\times 25$.

Table 2 Locations and one-LOD support intervals of QTL for molar size (QTL-CS) and molar shape (QTL-SH)

\begin{tabular}{lllclcr}
\hline QTL & LOD & Proximal marker & Marker distance & Marker support interval & Additive effects & Dominance effects \\
\hline QTL-CS12 & $3.606^{* *}$ & D12Mit214 & 4 & D12Mit112+6-D12Mit214+4 & $0.0144^{* *}$ & -0.0004 \\
QTL-SH12 & $3.157^{* *}$ & D12Mit105 & 0 & D12Mit105+0-D12Mit105+14 & $0.0024^{* *}$ & $0.0040^{* *}$ \\
\hline
\end{tabular}

Abbreviations: LOD, logarithms of odd; QTL, quantitative trait loci.

Locations of the QTL and their support intervals are given in terms of the distance in cM from the nearest proximal marker. Additive and dominance effects were obtained from multiple regressions.

${ }^{* *} P<0.01$ in chromosome-wide significance tests.

Interval mapping of chromosome 12 also uncovered one QTL for molar shape (QTL-SH12) whose LOD score (3.157) reached $1 \%$ chromosome-wide significance (Table 2). For this $Q T L$, the $\|d\|$ value (0.0040) is greater than the $\|a\|$ value (0.0024), indicating overdominance. The additive effects are generally small and occur primarily on the second and third molars; specifically at landmarks 4-8 (see Figure 2). The largest additive effect is seen at landmark 7, which moves posteriorly. Dominance effects of QTL-SH12 are seen at most landmarks, with the greatest effects occurring on the second molar at landmarks 4,7 and 8. The effects of this QTL act primarily to shift landmarks 4 and 8 in an anterior direction and landmark 7 in a posterior direction.

No significant results were obtained in tests of the effects of TCDD on C12 QTL (including QTL-CS12 and QTL-SH12) for any of the molar traits. Therefore, there is no evidence that TCDD influences the expression of any of the C12 QTL on the molar traits.

\section{Epistasis analysis}

None of the LOD scores testing for overall epistasis between QTL-SH11 and each of the two QTL discovered on $\mathrm{C} 12$ reached significance for any of the molar traits. However, a single dominance by additive $(d a)$ epistatic component for the QTL-SH11 by QTL-CS12 interaction was significant $(P=0.005)$ for molar centroid size. This suggests that at least one of the components of epistatic interaction between these two QTL affects molar size. We tested whether this $d a$ interaction differed in the TCDD and control environments, as previously explained, and in fact this test also reached significance $(P=0.007)$. Thus the epistatic interaction between the two QTL affecting molar size varies depending on whether the maternal environment did or did not contain TCDD.
The effects of the dominance by additive epistatic interactions of these two QTL in the two environments are depicted in Figure 3. One noticeable difference in the epistatic effect of the two QTL is seen in the interaction of the BB genotype of QTL-SH11 with QTL-CS12. As shown, the average molar size of QTL-SH11 BB individuals increases with TCDD exposure when they are heterozygous at the QTL-CS12 locus, but decreases when they are homozygous for either allele at that locus. In addition, QTL-SH11 demonstrates underdominance (heterozygotes less than either homozygote) for QTL-CS12 $\mathrm{AA}$ individuals in the control environment but not in the TCDD environment.

\section{Discussion}

\section{Evaluation of TCDD effects}

One of the primary goals of this study was to evaluate the effects of TCDD exposure on FA of molar size and shape in mice. As FA is thought to be a sensitive indicator of DI and TCDD is known to be a potent environmental toxicant, we hypothesized that in utero exposure to TCDD would reduce DS in mice and that this would be reflected in higher molar FA in the exposed mice. Significantly greater molar shape FA was detected in TCDD-treated mice compared to untreated controls, although the magnitude of this effect was small.

Allen and Leamy (2001) did not detect differences in mandible FA in this same population of mice, casting doubt on the usefulness of FA as a universal measure of DI. However, it has been increasingly recognized that the effects of various stressors on DS are trait specific (Indrasamy et al., 2000). The significant effect of TCDD on FA of molars but not mandibles may have been a consequence of the well-documented sensitivity of tooth 

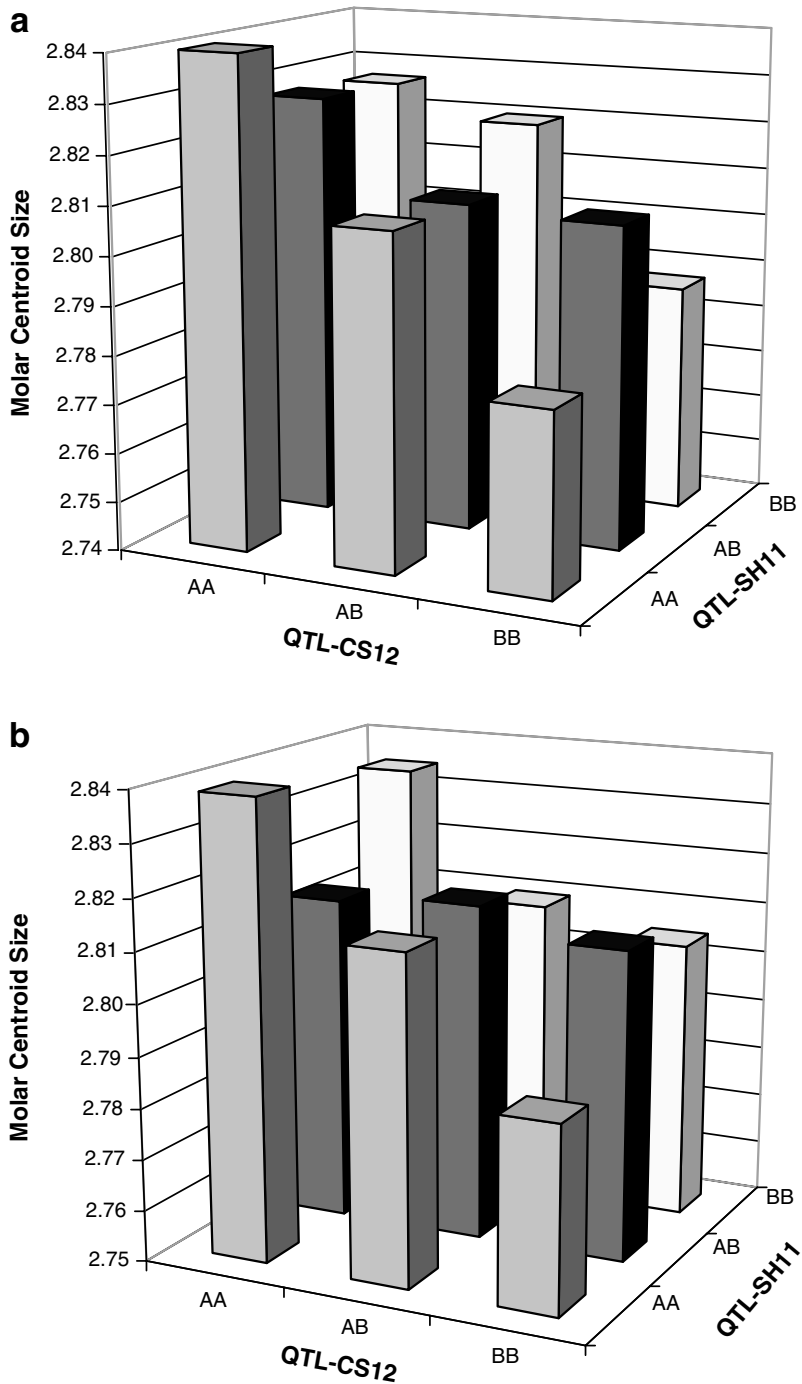

Figure 3 Dominance-by-additive epistatic interaction between QTL-SH11 and QTL-CS12 affecting molar centroid size in TCDD (a) and control (b) environments.

development to TCDD (see Alaluusua et al., 1993; Partanen et al., 1998).

Bone's ability to remodel after initial development, particularly with heavy use (Nomura and TakanoYamamoto, 2000; Garland and Freeman, 2005), may make mandibles (and other such bones) less suitable traits for the assessment of FA than teeth (Saunders and Mayhall, 1982), which do not change after their initial formation. It is possible that the subtle effects on FA that occur during development are masked by later remodeling, whereas gross changes, such as changes in size and shape, are not as readily eliminated. Changes in FA could also be obscured if the normal variance between sides is sufficiently high owing to remodeling that the power to detect the more subtle changes caused by low levels of TCDD is reduced. This may explain why Leamy et al. (1999a) did not detect differences in mandible FA in mice treated with methoxyclor despite detecting changes in the size of various mandible traits. Pankakoski et al. (1992) were able to detect increased mandible FA in shrews owing to heavy metal exposure, but these animals were taken as adults from an area of heavy metal contamination and presumably had been continually exposed to this stress throughout their lives. Other stressors which lead to detectable changes in mandible FA, such as inbreeding (e.g., see Leamy et al., 2001), are also experienced throughout life and, therefore may have greater potential to significantly affect FA of skeletal traits despite remodeling.

The detrimental effect of TCDD on tooth development has been well demonstrated (Alaluusua et al., 1993; Kattainen et al., 2001), so it is surprising that we failed to detect changes in molar size and shape with TCDD exposure. Perhaps the date of dosing used in this study was less suitable for causing detectable changes in overall molar size and shape than it was for mandible traits (Allen and Leamy, 2001; Miettinen et al., 2002). Whereas mandible formation has already begun by GD9 when these mice were dosed, tooth morphogenesis does not begin until GD11, and TCDD appears to particularly affect tooth development in mice during a sensitive period from GD12 to GD15 (Partanen et al., 2004). Abbott et al. (1994) showed that there is a significant reduction in TCDD levels in the embryos of exposed mothers after only 3 days, so perhaps too little TCDD remained in the developing embryos of our mice during the molar formation period.

Our results indicate that molar size or shape FA is not a particularly sensitive measure of developmental stress caused by TCDD exposure. Size FA was not at all affected by TCDD and shape FA was only slightly affected, at least at the levels we used. These dosage levels of TCDD were chosen because they are within the range of exposure levels that can occur with chronic exposure in natural populations, yet are lower than those generally expected to have a significant effect on survival (Birnbaum, 1994). For example, the lowest observable adverse effect level for prenatal mortality in mice owing to TCDD (Peterson et al., 1993) was 24 times $(24 \mu \mathrm{g} / \mathrm{kg})$ the highest level used in this study, whereas the mouse $\mathrm{LD}_{50}$ for oral exposure (Birnbaum 1994) is higher still (114-280 $\mu \mathrm{g} / \mathrm{kg})$. In any event, the change in shape FA that we observed is far more subtle than the gross morphological (and possibly detrimental) changes in symmetry observed in the brains of birds exposed to TCDD in ovo (Henshel et al., 1997).

\section{QTL analysis}

A second major goal of this study was to evaluate the effects of TCDD on gene expression. Because Davis et al. (2002) were able to detect a genotype $\times$ environment interaction for mandible shape FA in these mice, we hypothesized that we would find similar interactions affecting FA in one or more of the molar traits. But as will be recalled, we did not find any such interaction, suggesting that genes in the regions tested are not behaving differently in their effects on molar FA in the control compared to the TCDD environment. It seems unlikely that this result was due to insufficient statistical power because the same population of mice was used in both studies, and we were able to detect significant FA differences. It is of course possible that the effects of TCDD on molar shape FA are mediated by genes located elsewhere in the genome.

Perhaps a more likely explanation for our failure to detect an interaction affecting molar FA is that the gene 
identified in the study by Davis et al. (2002) was not a gene for FA per se, but was instead a gene for mandible development that was affected by TCDD (Klingenberg 2003). Because TCDD acts on gene regulatory elements via the AHR to alter the expression of genes at a number of loci (Birnbaum, 1995), a particular gene might exhibit a genotype $\times$ environment interaction affecting $F A$ if TCDD changes the way that the gene is expressed, causing greater stochastic variation than when TCDD is not present (Klingenberg, 2003). Such genes, if they exist, could also epistatically affect FA, and therefore still might not be detected in this analysis (Klingenberg, 2003; Leamy, 2003). In fact, there is evidence for epistatic interactions controlling FA in molar and mandible traits (Leamy et al., 2005). It seems likely that genes involved in mediating TCDD's effects on FA might also act in a primarily epistatic manner. Although no epistatic interactions were found to affect FA in this analysis, this is not surprising considering the high power required to detect such interactions and the limited amount of the genome mapped in this study.

Unlike genes for FA, genes for tooth and bone traits appear to be ubiquitous within the mouse genome (Leamy et al., 1999b; Workman et al., 2002). Even with our limited sample of the genome, we were able to detect a QTL for molar size as well as two QTL for molar shape in this analysis. The QTL for molar shape linked to D11Mit258 was of particular interest because Davis et al. (2002) found a QTL for mandible shape linked to this same locus that exhibited both a genotype $\times$ environment interaction and an epistatic effect with a gene on chromosome 12. Although we did not find a similar genotype $\times$ environment interaction for either molar size or molar shape in this analysis, we did find an epistatic effect on molar size generated by genes on C11 and C12. In addition, we detected the presence of an epistasis $\times$ environment interaction affecting molar centroid size. Although this interaction was only marginally significant, it suggests that TCDD has affected the way these genes interact. This is an interesting result because it demonstrates the type of complex interaction that might be prevalent among genes involved in controlling the development of quantitative traits, and perhaps the symmetry of bilateral traits (Klingenberg, 2003; Leamy, 2003). Our ability to detect such an interaction with only minimal TCDD exposure may explain the apparent sensitivity of development to the effects of TCDD and the wide range of effects it elicits.

\section{Conclusions}

The presence of significantly greater FA in molar shape (but not size) in the TCDD-treated group compared to the control group suggests that TCDD may have an effect on DS. The effect detected is very small, however, and thus we must conclude that FA in these tooth traits is not a sensitive indicator of TCDD's effects at the dosages we used. Although we were unable to locate any genes interacting directly with TCDD to affect FA, we only evaluated a small portion of the genome. A similar analysis performed on the whole genome might produce more gene $\times$ TCDD and/or epistasis $\times$ TCDD interactions affecting these molar traits. If so, this would help us better understand the pathway through which TCDD exerts its effect. Further, a full chromosome analysis of chromosome 11 might enable us to suggest candidate genes involved in the interaction with TCDD detected in this study.

\section{Acknowledgements}

It is a pleasure to thank Amir Motameni, Megan Chryst, Amanda Mims and Stacey Hartberger for their help in genotyping, Susan Peters, Christian Klingenberg and Yvette Huet-Hudson for suggestions on earlier versions of this paper, and two anonymous reviewers for excellent suggestions for revision. This research was supported in part by funds provided by the University of North Carolina at Charlotte.

\section{References}

Abbott BD, Perdew GH, Birnbaum LS (1994). Ah receptor in embryonic mouse palate and effects of TCDD on receptor expression. Toxicol Appl Pharmacol 126: 16-25.

Alaluusua S, Lukinmaa PL, Pohjanvirta R, Unkila M, Tuomisto J (1993). Exposure to 2,3,7,8-tetrachlorodibenzo- $p$-dioxin leads to defective dentin formation and pulpal perforation in rat incisor tooth. Toxicology 81: 1-13.

Allen DE, Leamy LJ (2001). 2,3,7,8-Tetrachlorodibenzo- $p$-dioxin affects size and shape, but not asymmetry of mandibles in mice. Ecotoxicology 10: 167-176.

Birnbaum LS (1994). The mechanism of dioxin toxicity: relationship to risk assessment. Environ Health Perspect 102: 157-167.

Birnbaum LS (1995). Developmental effects of dioxins. Environ Health Perspect 103: 89-94.

Cheverud JM (2001). A simple correction for multiple comparisons in interval mapping genome scans. Heredity 87: 52-58.

Cobourne MT, Sharpe PT (2003). Tooth and jaw: molecular mechanisms of patterning in the first branchial arch. Arch Oral Biol 48: 1-14.

Davis CR, Allen DE, Leamy LJ (2002). 2,3,7,8-Tetrachlorodibenzo-p-dioxin: its effect on genes for mandible traits in mice. Environ Toxicol Pharmacol 12: 43-53.

Garland Jr T, Freeman PW (2005). Selective breeding for high endurance running increases hindlimb symmetry. Evolution 59: 1851-1854.

Haley CS, Knott SA (1992). A simple regression technique for mapping quantitative trait loci in line crosses using flanking markers. Heredity 69: 315-324.

Henshel DS, Martin JW, DeWitt JC (1997). Brain asymmetry as a potential biomarker for developmental TCDD intoxication: a dose-response study. Environ Health Perspect 105: 718-725.

Indrasamy H, Woods RE, McKenzie JA, Batterham P (2000). Fluctuating asymmetry of specific bristle characters in Notch mutants of Drosophila melanogaster. Genetica 109: 151-159.

Kattainen H, Tuukkanen J, Simanainen U, Tuomisto JT, Kovero O, Lukinmaa PL et al. (2001). In utero/lactational 2,3,7,8tetrachlorodibenzo- $p$-dioxin exposure impairs molar tooth development in rats. Toxicol Appl Pharmacol 174: 216-224.

Kaufman MH (1992). The Atlas of Mouse Development. Academic Press: San Diego.

Klingenberg CP (2003). A developmental perspective on developmental instability: theory, models, and mechanisms. In: Polak $\mathrm{M}$ (ed). Developmental Instability: Causes and Consequences. Oxford University Press: New York. pp 14-34.

Klingenberg CP, Leamy LJ, Routman EJ, Cheverud JM (2001). Genetic architecture of mandible shape in mice: effects of quantitative trait loci analyzed by geometric morphometrics. Genetics 157: 785-802.

Klingenberg CP, McIntyre GS (1998). Geometric morphometrics of developmental instability: analyzing patterns of fluctuating asymmetry with Procrustes methods. Evolution 52: 1363-1375. 
Klingenberg CP, Monteiro LR (2005). Distances and directions in multidimensional shape spaces: implications for morphometric applications. System Biol 54: 678-688.

Leamy L (2003). Dominance, epistasis and fluctuating asymmetry. In: Polak $\mathrm{M}$ (ed). Developmental Instability: Causes and Consequences. Oxford University Press: New York. pp 142-156.

Leamy LJ, Doster MJ, Huet-Hudson YM (1999a). Effects of methoxychlor on directional and fluctuating asymmetry of mandible characters in mice. Ecotoxicology 8: 63-71.

Leamy LJ, Klingenberg CP (2005). The genetics and evolution of fluctuating asymmetry. Annu Rev Ecol Evol Syst 36: 1-21.

Leamy LJ, Meagher S, Taylor S, Carroll L, Potts WK (2001). Size and fluctuating asymmetry of morphometric characters in mice: their associations with inbreeding and the $t$-haplotype. Evolution 55: 2333-2341.

Leamy LJ, Routman EJ, Cheverud JM (1999b). Quantitative trait loci for early and late developing skull characters in mice: a test of the genetic independence model of morphological integration. Am Nat 153: 201-214.

Leamy LJ, Workman MS, Routman EJ, Cheverud JM (2005). An epistatic genetic basis for fluctuating asymmetry of tooth size and shape in mice. Heredity 94: 316-325.

Lincoln S, Daly M, Lander E (1992). Constructing Genetic maps with MAPMAKER/EXP 3.0, 3rd edn. Whitehead Institute: Cambridge, MA.

Lynch M, Walsh B (1998). Genetics and Analysis of Quantitative Traits. Sinauer Associates: Sunderland.

Miettinen HM, Alaluusua S, Tuomisto J, Viluksela M (2002). Effect of in utero and lactational 2,3,7,8-tetrachlorodibenzo- $p$ dioxin exposure on rat molar development: the role of exposure time. Toxicol Appl Pharmacol 184: 57-66.

Moore JA, Gupta BN, Zinkl JG, Vos JG (1973). Postnatal effects of maternal exposure to 2,3,7,8-tetrachlorodibenzo- $p$-dioxin (TCDD). Environ Health Perspect 5: 81-85.

Nomura S, Takano-Yamamoto T (2000). Molecular events caused by mechanical stress in bone. Matrix Biol 19: 91-96.
Palmer AR (1994). Fluctuating asymmetry analyses: a primer. In: Markow TA (ed). Developmental Instability: Its Origins and Evolutionary Impacts. Kluwer Academic Publishers: Dordrecht. pp 335-364.

Palmer AR, Strobeck C (2003). Fluctuating asymmetry analyses revisited. In: Polak $\mathrm{M}$ (ed). Developmental Instability: Causes and Consequences. Oxford University Press: New York. pp 279-319.

Pankakoski E, Koivisto I, Hyvärinen H (1992). Reduced developmental stability as an indicator of heavy metal pollution in the common shrew Sorex araneus. Acta Zool Fennica 191: 137-144.

Partanen AM, Alaluusua S, Miettinen PJ, Thesleff I, Tuomisto J, Pohjanvirta R et al. (1998). Epidermal growth factor receptor as a mediator of developmental toxicity of dioxin in mouse embryonic teeth. Lab Invest 78: 1473-1481.

Partanen AM, Kiukkonen A, Sahlberg C, Alaluusua S, Thesleff I, Pohjanvirta R et al. (2004). Developmental toxicity of dioxin to mouse embryonic teeth in vitro: arrest of tooth morphogenesis involves stimulation of apoptotic program in the dental epithelium. Toxicol Appl Pharmacol 194: 24-33.

Peterson RE, Theobald HM, Kimmel GL (1993). Developmental and reproductive toxicity of dioxins and related compounds: cross-species comparisons. Crit Rev Toxicol 23: 283-335.

Saunders SR, Mayhall JT (1982). Fluctuating asymmetry of dental morphological traits: new interpretations. Hum Biol 54: 789-799.

Sokal RR, Rohlf JF (1995). Biometry, 3rd edn. WH Freeman: New York.

Whitlock JP (1999). Inductionof cytochrome P4501A1. Annu Rev Pharmacol Toxicol 39: 103-125.

Wolf JB, Leamy LJ, Routman EJ, Cheverud JM (2005). Epistatic pleiotropy and the genetic architecture of covariation within early- and late-developing skull trait complexes in mice. Genetics 171: 683-694.

Workman MS, Leamy LJ, Routman EJ, Cheverud JM (2002). Analysis of quantitative trait locus effects on the size and shape of mandibular molars in mice. Genetics 160: 1573-1586. 